\title{
OCT based Comparison of RNFL Thickness in Low to Moderate Myopic Eyes with NORMAL and Raised IOP
}

\author{
Snigdha Sen ${ }^{1}$, Himanshu Yadav², Tirupati Nath ${ }^{3}$, Arpita Gupta ${ }^{4}$
}

\begin{abstract}
Purpose: To compare Retinal Nerve Fibre Layer (RNFL) thickness with Optical Coherence Tomography (OCT) in low to moderate myopic eyes with normal \& raised Intra Ocular Pressure (IOP) without any other glaucomatous changes. Methods: A Total of 60 low to moderate myopes (spherical equivalent [SE] between -6.0D \& -0.5D) with 30 subjects with normal IOP $(\leq 21 \mathrm{mmHg}$ ) and 30 with raised corrected IOP $(\geq 2 \mathrm{mmHg}$ ) were included in this cross-sectional study. Three circular scans were obtained for each eye at diameter of $3.4 \mathrm{~mm}$ around the optic disc using RTVue OCT. A single index of average RNFL thickness throughout 360 degree was obtained. The mean RNFL of (n=60) eyes with raised IOP was compared with age matched randomly selected (n=60) eyes with normal IOP. Results: Mean RNFL was significantly thinner in eyes with raised IOP than in eyes with normal IOP, $91.01 \pm 11.95$ and $101.52 \pm$ 10.13 respectively. Conclusion: These findings suggest that quantitative differences in RNFL thickness exist between age-matched low to moderate myopic eyes with normal and raised IOP. Thus, all myopic individuals with raised IOP should be considered for OCT to assess for damage of RNFL, as they are at risk of developing glaucoma in future.
\end{abstract}

Keywords: RNFL thickness, myopia, intraocular pressure, OCT, glaucoma

\section{Introduction}

OCT has allowed in vivo quantitative analysis of the peripapillary RNFL, and measuring the RNFL has been useful for diagnosing glaucoma. ${ }^{1}$ At present, there is large number of individuals suffering from myopia. Its prevalence has increased markedly in recent Years. ${ }^{2,3}$ It is well known that myopia is a risk factor of glaucoma and myopic fundus changes may complicate the diagnosis and treatment. Assessment of the peripapillary RNFL thickness has been an important approach for detecting structural damage in patients with myopia with raised IOP as a risk factor for glaucoma. ${ }^{4}$ As there is considerable evidence that RNFL loss precedes visual field loss ${ }^{5-9}$ and optic nerve head defects in patients with glaucoma, ${ }^{10}$ it is of interest to quantify this loss in patients who are at risk for developing glaucoma. Although previous studies have shown the utility of peripapillary RNFL measurements in glaucoma patients, little is known about comparison between RNFL thickness in myopic patients with increased IOP using OCT. In this study, we assessed RNFL thickness in low to moderate myopic eyes and compared it between two groups, with normal IOP and raised IOP using the commercially available RTVue OCT version 6.3.

\section{Material and Methods}

Sixty low to moderate myopic (SE between -6.0D \& -0.5D) individuals ranging in age from 20 to 60 years were recruited in this study and were divided into 2 groups: Low to moderate myopic $(n=60)$ eyes with normal IOP $(\leq 21$ $\mathrm{mmHg})$ as (GROUP-I) and low to moderate myopic $(\mathrm{n}=60)$ eyes with raised IOP ( $\geq 22 \mathrm{mmHg}$ ) as (GROUP-II). All those individuals with age group: 20-60 years, BCVA 20/40 or more, Low-to-moderate myopia (SE between $-6.0 \mathrm{D}$ and $-0.5 \mathrm{D})$, without significant pathological fundus changes, Normal appearing optic discs, Open angles on gonioscopy and No glaucomatous changes in visual field were included in the study. While, individuals with Secondary glaucomas (eg. traumatic, inflammatory, neovascular), Advanced glaucomatous alterations, History of intraocular diseases(eg. inflammation, tumor), Complicated intraocular surgery, Advanced cases of cataract, Coexisting retinal diseases, Other diseases affecting VF (pituitary lesion, AIDS) were excluded from the study.

The study was complied with the tenets of the Declaration of Helsinki. Informed consent was obtained from all participants. All eyes underwent Comprehensive ophthalmological examination, Visual acuity, Refraction, Intraocular pressure measurement with goldmann applanation tonometer, Gonioscopy, Direct and indirect ophthalmoscopy, Stereoscopic fundus evaluation on the slit lamp using a 90-D lens, Visual fields using 30-2 SITA standard full threshold programme on Humphrey Field analysar perimeter (Carl Zeiss Meditec, Inc, Dublin,CA), OCT analysis for central corneal thickness and retinal nerve fibre layer thickness measurement.

OCT was performed with RTVue OCT version 6.3. All OCT scans were acquired by an experienced OCT operator. The investigator measuring the OCT parameters were masked to the patient diagnosis. The operator centered the circular scan on the optic nerve head (ONH) while the eye was fixated. Average measurements of three sequential circular scans of diameter $3.4 \mathrm{~mm}$ centered on the optic disc were recorded. Parameters including average RNFL thickness and superior, nasal, inferior and temporal quadrant thickness and for each of the 12 o'clock-hour positions were generated automatically in the analysis report.

The superior clock hour was 12 o'clock. The others were plotted in a clockwise direction for a right eye and counter clockwise for a left eye. RNFL thicknesses in the normal range were represented by green backgrounds, those that were abnormal at the $5 \%$ level were represented by yellow backgrounds, and those that were abnormal at the $1 \%$ level were represented by red backgrounds. All scans were acquired 3 times during the same day, with short breaks 


\section{International Journal of Science and Research (IJSR) \\ ISSN (Online): 2319-7064 \\ Index Copernicus Value (2013): 6.14 | Impact Factor (2014): 5.611}

between each measurement and an average of the 3 readings was computed in each case. Only good quality OCT scans were included in the final analysis. The comparison of RNFL thickness between two groups was done using unpaired student-t test.

\section{Results}

This study comprised of 120 eyes of 60 patients. Patients were categorized into two groups: Low to moderate myopic $(n=60)$ eyes with normal IOP $(\leq 21 \mathrm{mmHg})$ as (GROUP-I) and low to moderate myopic $(n=60)$ eyes with raised IOP $(\geq$ 22mmHg) as (GROUP-II). There were 34 males and 26 females. The mean age was 31.89 \pm 6.21 years (range 20-60 years), mean spherical equivalent was $-2.28 \pm 2.33 \mathrm{D}$ (range 0.5 to $-6.0 \mathrm{D})$.

With increasing age, the RNFL thickness was found to be decreasing and the result was statistically significant ( $p$ value $<0.05$ ) as shown in TABLE-1. However, the spherical equivalent did not show any differences in RNFL thickness. After the comparison of the RNFL thickness, we found significant differences between groups, which showed that average, superior, nasal, inferior, and temporal RNFL thickness was significantly reduced in GROUP-II when compared with GROUP-I and the difference was statistically significant ( $p$ value $<0.05$ ) as shown in TABLE-2.

\section{Discussion}

However, The current study demonstrates that OCT measures the RNFL as thinner in older persons, with a decline of approximately $2 \mu \mathrm{m}$ per decade. Because there have been no OCT longitudinal studies of RNFL thickness in healthy individuals, we make the assumption that a crosssectional analysis is a good surrogate for change over time in an individual. In contrast to previous studies using oldergeneration OCT, We evaluated peripapillary RNFL thickness in this study based on RTVue OCT and found that RNFL thickness decreased as the age advances. Several earlier studies have shown a decrease in RNFL thickness with age by OCT or in RNFL axons by histological analysis. ${ }^{11,12}$ A third histological study failed to find any difference in the number of axons between older and younger individuals. ${ }^{13}$ Conflicting results in histological studies may be because these studies look at relatively few subjects and there is a large variation in the number of RNFL axons in normal individuals, between 7,00,000 and 1.4 million.

$\mathrm{SE}$ in the study group is between -0.5 to $-3.0 \mathrm{D}$ for low myopic eyes and -3.0 to $-6.0 \mathrm{D}$ for eyes with moderate myopia. In our study RNFL thickness did not vary much with such differences. Several previous studies has also suggested that no significant correlation is associated between myopia and RNFL thickness. ${ }^{14}$ However, these studies may have been limited by the poorer resolution of earlier generation OCT and confocal laser devices which had lower sensitivity. Using $3^{\text {rd }}$ generation OCT3, it has been reported that RNFL thickness increased with increasing hyperopia (1.7 microns per diopter) in children (91\% Hispanic, mean age 10 years, range 4-17). Alternatively, significant decrease in RNFL thickness with increasing axial length in myopia has been reported. However, its decreasing ratios varied (2-7 microns per $\mathrm{mm}$ ) in reports; as such, its changes of RNFL thickness may depend exclusively on different races and age distributions. ${ }^{15}$

It is generally known that tilted disc is more prevalent in myopic eyes, and thus this distortion of the optic nerve fibers at the myopic disc may cause a mechanical stress toward the optic nerve fibers, eventually leading to the glaucomatous optic neuropathy.

However, our present data may not support this mechanical theory in low to moderate myopic eyes, but it may hold true for high myopic eyes.

This study quantified and compared RNFL thickness in myopic eyes with raised IOP to myopic eyes with normal IOP by using OCT. There was considerable within-group variability in measured RNFL thickness. Average RNFL was significantly thinner in group-2 eyes than in group-1 eyes. Specifically, RNFL was significantly thinner in the inferior and nasal quadrants.

Similar findings have been reported elsewhere using different evaluative techniques. Using stereo photogrammetric measures, Schwartz and Takamoto showed a significant decrease in RNFL thickness of about $18 \%$ in OHT eyes compared with normal eyes. Similar results were obtained by Tjon-Fo-Sang et al. ${ }^{16}$ also using scanning laser polarimetry. Finally, using computerized image analysis, Poinoosawmy $\mathrm{D}^{17}$ found a significant decrease in retinal height (an indirect measure of RNFL thickness) of almost $30 \%$ in OHT eyes compared with normal eyes.

As in this study, the latter 3 studies mentioned revealed a significant thinning of RNFL in the inferior quadrant in OHT eyes compared with normal eyes.

Since most of the studies are conducted in Ocular hypertensive (OHT) eyes, and none till date is done on myopic population, so this study gives us a new approach to evaluate myopic population with raised intraocular pressure for structural damage in RNFL via OCT. This approach may halt the further damage which may progress to glaucoma in later stage.

Table 1: Mean Retinal Nerve Fiber Layer (RNFL) Thickness Stratified by Age Category

\begin{tabular}{|c|c|c|c|}
\hline $\begin{array}{c}\text { age category } \\
\text { (years) }\end{array}$ & $\begin{array}{c}\text { no. of } \\
\text { subjects } \\
(\%)\end{array}$ & $\begin{array}{c}\text { Mean RNFL } \\
\text { Thickness }(\mu \mathrm{m}) \pm \\
\text { Standard Deviation }\end{array}$ & $\begin{array}{c}\text { 95\% Confidence } \\
\text { Interval }\end{array}$ \\
\hline $20-30$ & 31.66 & $118.7 \pm 9.7$ & $115.2-121.2$ \\
\hline $31-40$ & 28.33 & $114.7 \pm 10.4$ & $112.7-117.8$ \\
\hline $41-50$ & 21.66 & $103.9 \pm 12.2$ & $99.1-107.7$ \\
\hline $51-60$ & 18.33 & $99.4 \pm 12.7$ & $96.4-102.3$ \\
\hline
\end{tabular}




\section{International Journal of Science and Research (IJSR) \\ ISSN (Online): 2319-7064}

Index Copernicus Value (2013): 6.14 | Impact Factor (2014): 5.611

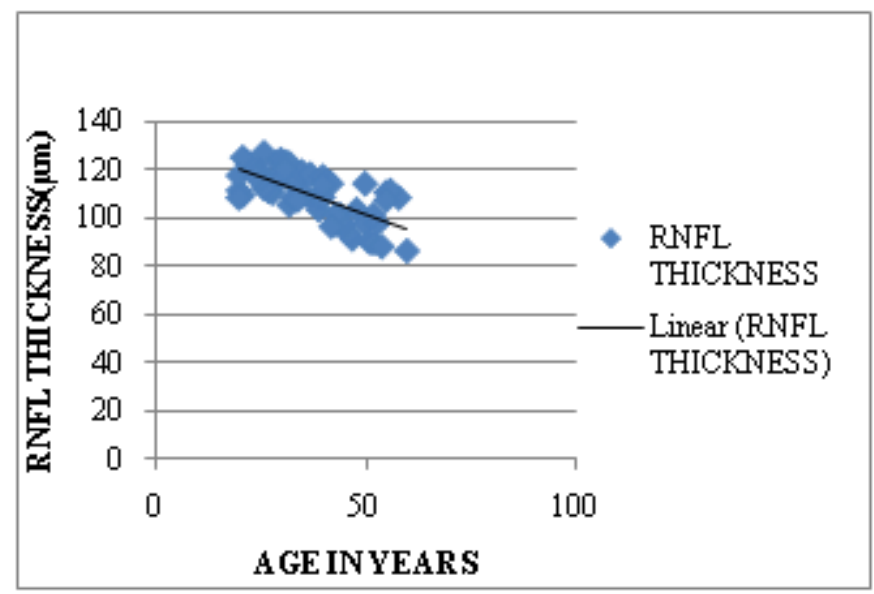

Table 2: RNFL Thickness Comparison between Groups

\begin{tabular}{|l|l|l|}
\hline RNFL PARAMETER & GROUP-1 $(\mu \mathrm{m})$ & GROUP-2 $(\mu \mathrm{m})$ \\
\hline $\begin{array}{l}\text { Average } \\
\text { 95\% CI }\end{array}$ & $\begin{array}{l}101.52 \pm 10.13 \\
98.58-104.47\end{array}$ & $\begin{array}{l}91.01 \pm 11.95 \\
87.97-98.06\end{array}$ \\
\hline $\begin{array}{l}\text { Temporal average } \\
\text { Mean } \pm \text { SD }\end{array}$ & $\begin{array}{l}128.73 \pm 13.15 \\
124.91-132.55\end{array}$ & $\begin{array}{l}117.0 \pm 15.02 \\
110.66-122.34\end{array}$ \\
\hline $\begin{array}{l}\text { Superior average } \\
\text { Mean } \pm \text { SD }\end{array}$ & $123.21 \pm 15.55$ & $110.25 \pm 18.29$ \\
95\% CI & $118.69-127.72$ & $102.53-117.97$ \\
\hline $\begin{array}{l}\text { Nasal average } \\
\text { Mean } \pm \text { SD } \\
\text { 95\% CI }\end{array}$ & $87.85 \pm 17.40$ & $77.83 \pm 20.94$ \\
\hline $\begin{array}{l}\text { Inferior average } \\
\text { Mean } \pm \text { SD }\end{array}$ & $62.80-92.91$ & $68.99-86.68$ \\
95\% CI & $61.66-70.17$ & $66.79 \pm 8.65$ \\
\hline
\end{tabular}

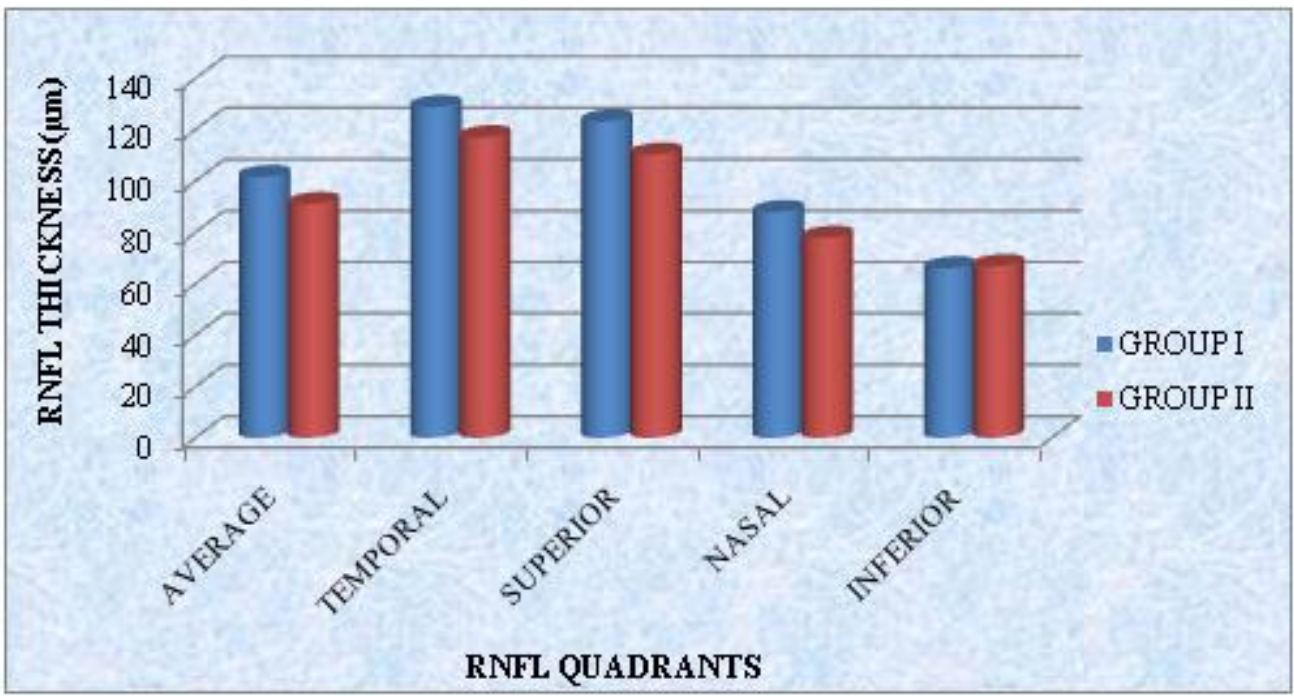

\section{References}

[1] Huang ML and Chen HY, 2005: Parikh RS et. al. 2007

[2] Lin LL, Shih YF, Hsiao CK, Chen CJ, Lee LA, Hung PT. Epidemiologic study of the prevalence and severity of myopia among school children in Taiwan in 2000. 2001;100(10):684-691

[3] Wong TY, Foster PJ, Hee J, Ng TP, Tielsch JM, Chew SJ, Johnson GJ, Seah SK. Prevalence and risk factors for refractive errors in adult Chinese in Singapore. 2000;41(9):2486-2494

[4] Quigley HA, Addicks EM. Quantitative studies of retinal nerve fiber layer defects. 1982;100(5):807-814

[5] Sommer AKatz JQuigley HA et al. Clinical detectable nerve fiber atrophy precedes the onset of glaucomatous field loss. Arch Ophthalmol. 1991;1097783

[6] Quigley HAAddicks EMGreen WR Optic nerve damage in human glaucoma, III: quantitative correlation of nerve fiber loss and visual field defect in glaucoma, ischemic neuropathy, papilledema, and toxic neuropathy. Arch Ophthalmol. 1982;100135- 146

[7] Quigley HADunkelberger GRGreen WR Retinal ganglion cell atrophy correlated with automated perimetry in human eyes with glaucoma. Am J Ophthalmol. 1989;107453- 464
[8] Quigley HAEnger CKatz J et al. Risk factors for the development of glaucomatous visual field loss in ocular hypertension. Arch Ophthalmol. 1994;112644- 649

[9] Sommer AMiller NRPollack IMaumenee AEGeorge $\mathrm{T}$ The nerve fiber layer in the diagnosis of glaucoma. Arch Ophthalmol. 1977;952149- 2156

[10] Airaksinen PJAlanko HI Effect of retinal nerve fibre loss on the optic nerve head configuration in early glaucoma. Graefes Arch Exp Ophthalmol. 1983;220193- 196

[11] Balazsi AG, Rootman J, Drance SM, et al. The effect of age on the nerve fiber population of the human optic nerve. Am J Ophthalmol. 1984;97:760-6.

[12] Johnson BM, Miao M, Sadun AA. Age-related decline of human optic nerve axon populations. Age. 1987;10:5-9.

[13] Repka MX, Quigley HA. The effect of age on normal human optic nerve fiber number and diameter. Ophthalmology. 1989;96:26-32.

[14]BowdC, ZangwillLM, BlumenthalEZ, et al. Imaging of the optic disc and retinal nerve fiber layer: the effects of age, optic disc area, refractive error, and gender. $J$ Opt Soc Am A Opt Image Sci Vis. 2002;19:197-207.

[15] HohST, LimMC, SeahSK, et al. Peripapillary retinal nerve fiber layer thickness variations with myopia. Ophthalmology. 2006;113:773-777.

[16] Tjon-Fo-Sang MJ, de Vries J, Lemij HG. Measurement by nerve fiber analyzer of retinal nerve fiber layer 


\section{International Journal of Science and Research (IJSR) \\ ISSN (Online): 2319-7064}

Index Copernicus Value (2013): 6.14 | Impact Factor (2014): 5.611

thickness in normal subjects and patients with ocular hypertension. Am J Ophthalmol. 1996;122:220-7.

[17] Poinoosawmy D, Fontana L, Wu JX, et al. Variation of nerve fibre layer thickness measurements with age and ethnicity by scanning laser polarimetry. $\mathrm{Br} J$ Ophthalmol. 1997;81:350-4. 